\title{
Correction to: Superplasticity: Recent Approaches and Trends
}

Deepika M. Harwani, Vishvesh J. Badheka, and Vivek Patel

Correction to:

Chapter "Superplasticity: Recent Approaches and Trends" in: A. K. Parwani et al. (eds.),

Recent Advances in Mechanical Infrastructure, Lecture Notes in Intelligent Transportation and Infrastructure, https://doi.org/10.1007/978-981-33-4176-0_33

In the original version of the book, in Chapter "Superplasticity: Recent Approaches and Trends" the author name "Vivek K. Patel" name is replaced with "Vivek Patel". The chapter and book have been updated with the change. 